\title{
Baroreceptor Stimulation Enhanced Nitric Oxide Vasodilator Responsiveness, a New Aspect of Baroreflex Physiology
}

Running head: Arterial baroreflex and NO vasodilation

\author{
Juraj Gmitrov ${ }^{1,2}$ \\ ${ }^{1}$ National Institute of Public Health, Department of Environmental Health, Tokyo 108, \\ Japan \\ ${ }^{2}$ Pro Vitae Hospital, Diabetology Clinic, Nemocnicna 33, Gelnica, 05601, Slovak Republic \\ ${ }^{1}$ Institution where the experimental part of the study was made \\ ${ }^{2}$ Current affiliation
}

Address of Corresponding Author:

Juraj Gmitrov, MD, PhD

Visnova 6, Presov 080 01,

Slovak Republic

Phone: +421907506201

e-mail: gmitrovj@yahoo.com 


\begin{abstract}
Objective: Increasing evidence suggests nitric oxide (NO) deficit and baroreflex dysfunction to be characteristic for cardiovascular conditions even in preclinical stages of the disease. Sodium nitroprusside (SNP), a spontaneous NO-donor, vasodilatory effect was studied in conjunction with sinocarotid baroreceptor magnetic stimulation and potential implementation in NO deficiency states.

Methods: Mean femoral artery blood pressure (MAP), heart rate (HR) and ear lobe skin microcirculatory blood flow, measured by microphotoelectric plethysmogram (MPPG), were simultaneously recorded in conscious rabbits before and after 40-min sinocarotid baroreceptors exposure to $350 \mathrm{mT}$ static magnetic field $(\mathrm{SMF})$, generated by $\mathrm{Nd}_{2}-\mathrm{Fe}_{4}-\mathrm{B}$ alloy $(\mathrm{n}=8)$ or sham magnets ( $\mathrm{n}=8$, controls). Arterial baroreflex sensitivity (BRS) was measured by changes in HR and MAP $(\triangle \mathrm{HR} / \triangle \mathrm{MAP})$ after intravenous bolus injections of SNP and phenylephrine.

Results: The vasodilatory effect of SNP significantly increased after SMF exposure $\left(\mathrm{MPPG}_{\text {beforeSMF}}: 2.57 \pm 0.81 \mathrm{~V} v\right.$ s. $\left.\mathrm{MPPG}_{\mathrm{afterSMF}}: 7.82 \pm 1.61 \mathrm{~V}, \mathrm{p}=0.0001\right)$ and positively correlated with significant increase in BRS $(r=0.51, \mathrm{p}<0.01)$.

Conclusions: Baroreflex-mediated increment in vessels sensitivity to NO suggested to be a new mechanism in baroreflex physiology with potential implementation in cardiovascular conditions where NO deficit and autonomic dysfunction increases the risk of morbidity and mortality substantially.
\end{abstract}

Keywords: static magnetic field, baroreflex sensitivity, microcirculation, nitric oxide, vascular smooth muscle sensitivity to nitric oxide 


\section{List of abbreviations}

BRS, arterial baroreflex sensitivity

$\mathrm{BRS}_{\mathrm{Ni}}$, arterial baroreflex sensitivity tested by nitroprusside

$\mathrm{BRS}_{\mathrm{Ph}}$, arterial baroreflex sensitivity tested by phenyleprine

$\mathrm{HR}$, heart rate

MAP, mean femoral artery blood pressure

MPPG, microphotoelectric plethysmography

NO, nitric oxide

REC, rabbit ear chamber

SMF, static magnetic field

\section{Introduction}

Arterial baroreceptors play a key role in the operation of cardiovascular functions and in reflex regulation of blood pressure, located in the carotid sinuses and aortic arch, normally respond to stretch by initiating reflexes that promote parasympathetic and restrain sympathetic activities, significantly modifying heart rate, peripheral vasoconstriction and cardiac output. These powerful negative feedback responses result in hemodynamic stability protecting vessels and the heart from extensive blood pressure fluctuations and arrhythmias, adjusting peripheral tissues perfusion to meet the optimal metabolic demands (Chapleau and Abboud, 2004).

However, arterial baroreflex direct regulatory effect on microcirculatory blood flow is less explored. The microcirculatory network of the skin, like other body tissues, continuously exhibits vasomotion, rhythmic changes in diameter and flow reflecting central baroreflex-mediated neurogenic adjustments (Bernardi et al., 1997; Szili-Torok et al., 2002) and local intrinsic myogenic activity modulated in addition by a variety of endothelial vasoactive compounds including nitric oxide (NO) (Harrison and Cai, 2003; Napoli et al., 2006). NO is a highly reactive signaling molecule that has pivotal role in a spectrum of fundamental intracellular events that lead to vasorelaxation, inhibition of platelet aggregation, endothelial regeneration, suppression of abnormal proliferation of vascular smooth muscle cells and cardiovascular remodeling (Harrison and Cai, 2003). The clinical importance of the NO is so large that this gas suggested to be the most important affecting almost every aspect of cardiovascular physiology extended to metabolic 
disorders, insulin resistance and atherosclerosis (Harrison and Cai, 2003; Kim et al., 2006; Napoli et al., 2006). A mounting evidence indicates that NO has an outstanding modulatory effect on sympathetic and parasympathetic control of cardiovascular function at all levels of the autonomic regulation including central arterial baroreflex pathways (Schultz, 2009). However, no studies demonstrated arterial baroreflex feedback coupling with NO-mediated vasodilation which may have a particular clinical importance.

Previously we found in rabbits that a $350 \mathrm{mT}$ static magnetic field (SMF) when locally applied to sinocarotid triangles enhanced microvascular dilation and blood flow in the visualized cutaneous vasculature of the ear and that this effect significantly correlated with increase in baroreflex sensitivity (Gmitrov, 2007). To test hypothesis that the underlying mechanism lies in baroreflex modulatory effect on NO-dependent vasodilation the magnitude of the cutaneous vasodilator response to sodium nitroprusside, a spontaneous NO donor (Schröder, 2006), was determined in relation to increase in baroreflex sensitivity generated by carotid baroreceptors magnetic activation.

\section{Methods}

\section{Animals}

Sixteen experiments were performed in 11 adult males of Japanese domestic white rabbits, (oryctolagus cuniculus domesticus) weighing 3.5-4.2 kg (Nihon Ikagaku Shizai, Tokyo, Japan). Eight runs with the sinocarotid baroreceptors exposed to a SMF and 8 controls with sham magnets exposure. The time when these experiments were performed was chosen randomly from 10 a.m. to noon (local time). The rabbits were given free access to laboratory chow (RM4; Funabashi Nohjoh, Chiba, Japan) and tap water. They were housed in a room with a light/dark cycle of $12 \mathrm{~h}$, with a temperature of $22.5 \pm 0.5^{\circ} \mathrm{C}$ and a relative humidity of $50 \pm 5 \%$. All the procedures conform with the "Guide for the Care and Use of Laboratory Animals" published by the US National Institute of Health (NIH publication no. 85-23, revised 1996), and with guidelines of the Japanese national ethics committee. A polyvinyl catheter was introduced into the femoral artery to make hemodynamic recordings of mean femoral artery blood pressure (MAP) and heart rate (HR) resulting from blood pressure fluctuations. Rabbits were anesthetized for the operation with sodium pentobarbital (Nembutal Sodium Solution; Abbott Laboratories, Co., Ltd., Chicago, IL, USA), $30 \mathrm{mg} / \mathrm{kg}$, iv, supplemented later as needed to maintain deep 
anesthesia. The catheter was fed subcutaneously into the back of the animals, filled with $0.9 \%$ $\mathrm{NaCl}$ solution containing heparin $2000 \mathrm{U} \mathrm{ml} / 1$ and plugged with a polyvinyl plug. Catheters were flushed daily. An average of $1-2$ experiments were performed on each rabbit. One experiment was performed per day per animal, the first commencing $24 \mathrm{~h}$ after catheterization alternating sham and SMF experimental runs. The rabbits were not sacrificed at the end of the experiments.

\section{Measurement of skin microcirculatory blood flow}

Intravital microscopy using a rabbit ear chamber (REC) and microphotoelectric plethysmography (MPPG) were used to measure microcirculatory blood flow in the skin. A REC is a transparent disk (diameter: $6.4 \mathrm{~mm}$ ) of acrylic resin housing fully ingrown subcutaneous tissue with a microvascular net after around $6-8$ weeks following the installation, which is the subject of research (Fig. 1). MPPG is a simple, non-invasive technique used to analyze relative changes in peripheral circulation and is a modification of photo(electric) plethysmography. MPPG was developed to monitor temporal changes in microvascular blood flow in various cutaneous tissues. Microscopic images of the intravital microcirculation are displayed on a video monitor, and light absorbed by hemoglobin in the transparent microvascular net within the REC is detected directly using a photoconductive cell within the apparatus. Any microvascular alternations are then transformed to an electrical signal and traced as MPPG DC profiles and measured in volts (V), using a data recorder/analyzer. MPPG can be used to analyze whole microcirculatory systems or to study single vessels, and thus it allows accurate and precise investigation of even very delicate rhythmic fluctuations in microcirculation. MPPG profiles were taken continuously and the same microscopic magnifications were used (100x) during the entire experiment. Close positive correlations have already been found between MPPG profiles and a cross-sectional view of an artery (Okano and Ohkubo, 2001). Temperature was monitored by a thermistor (MGA-III-219, Nihon Kohden Co., Tokyo, Japan) placed in close proximity of the REC, and was held constant at $23^{\circ} \mathrm{C}$ during the entire experiment. Measurements using the MPPG were accompanied by concomitant intravital microscopy of the explored microvascular networks (Fig. 1). 


\section{Baroreflex assessment}

The arterial baroreflex was assessed by measuring arterial baroreflex sensitivity (BRS). BRS testing was performed using iv bolus injections of a small dose of a vasoactive drug (Soares et al., 2005). To elicit reflex changes in heart rate in response to changes in blood pressure, iv bolus injections of the rapid-acting vascular smooth muscle dilator, sodium nitroprusside $(\mathrm{Ni} ; 1.0,3.0$, 10 and $30 \mu \mathrm{g} \mathrm{kg}^{-1}$; sodium pentacyanonitrosylferrate (III) dihydrate; Wako Pure Chemical Industries Co., Ltd, Osaka, Japan), and the vasopressor phenylephrine (Ph; 0.3, 1.0, 3.0 and $10 \mu \mathrm{g}$ $\mathrm{kg}^{-1}$; neo-synesin; Kowa Pharmaceutical Co., Ltd, Tokyo, Japan) were performed. The test drugs were alternately injected iv in increasing doses; nitroprusside was administered first, then phenylephrine. Pressure ramps were applied at 3-min intervals for all doses, except for the last interval between nitroprusside $(30.0 \mu \mathrm{g} / \mathrm{kg})$ and phenylephrine $(10.0 \mu \mathrm{g} / \mathrm{kg})$, when the interval was $5 \mathrm{~min}$. After each bolus, the arterial blood pressure was allowed to return to its previous resting level before the next dose was given (Hof et al., 1991). The BRS was then calculated as the change in heart rate divided by the change in mean arterial blood pressure $(\triangle H R / \Delta M A P)$ in response to sodium nitroprusside $(3.0,10$ and $30 \mu \mathrm{g} \mathrm{kg}-1)$ and phenylephrine $(1.0,3.0$ and $10 \mu \mathrm{g}$ kg-1) administration (Omoniyi et al., 2000; Soares et al., 2005). Data were used only when animals were calm, resting, and undisturbed during the entire experimental run. Details on BRS testing and the MPPG technique can be found elsewhere (Gmitrov, 2007).

\section{Experimental procedure}

Rabbits were placed, prone, in a specially constructed metal drum from nonmagnetic material. The upper part of their noses and their jaws were rigidly inserted into a small ring attached to the anterior part of the drum, to stabilize the head, which sat outside the drum (Fig. 1). To minimize discomfort and immobilization stress, which would affect the blood pressure, heart rate and MPPG readings, the rabbits were sedated using pentobarbital iv infusion $\left(5 \mathrm{mg} \mathrm{kg}^{-1} \mathrm{~h}^{-1}\right)$ for 120 min lasting experiment. The rabbit's ear lobe was attached to the acrylic observing stage of the microscope, using plastic surgical adhesive tape strips to prevent any changes in the position of REC during the experiment.

Mean femoral artery blood pressure, heart rate and blood flow in the skin microvascular network of the ear lobe (MPPG) were simultaneously recorded using a polygraph (Nihon Kohden Co., Tokyo, Japan) and calculated using a data analyzer (DAA-110B; Kyowa Electronic 
Instruments Co., Ltd, Tokyo, Japan), with a sampling time of $200 \mathrm{~ms}$ (Fig. 1). Heart rate variability was quantified from femoral artery blood pressure fluctuations as the standard deviation (SD) of the average heart rate. The animals were randomized to sham vs. magnetic stimulation. Both arms had a baseline and treatment measurements. The first baseline, control measurement of BRS was made 15 min after the beginning of the experiment (Fig. 2). After 50 min, two sham or cylindrical $\mathrm{Nd}_{2}-\mathrm{Fe}_{4}-\mathrm{B}$ alloy magnets with magnetic field flux density of 500 $\mathrm{mT}$ (measured in the middle of the pole face surface), were carefully positioned with opposite poles under the right and left carotid sinus. Each magnet consisted of eight coin-shaped magnets, $23 \mathrm{~mm}$ in diameter and $5 \mathrm{~mm}$ in thickness, stuck together (Neomax, PIP; Tokyo Ltd, Tokyo, Japan). To avoid any pressure on the rabbits' neck, the magnets were held by manipulators at an angle of $110^{\circ}$ to each other, just touching the closely trimmed hair of the neck under the sinocarotid baroreceptors (Fig. 1). The field at the site of the baroreceptors, which were situated about $0.5 \mathrm{~cm}$ from the pole faces, was $350 \mathrm{mT}$. The SMF gradient was $30 \mathrm{~T} / \mathrm{m}$. After $40 \mathrm{~min}$ (i.e., 90 min after the beginning of the experiments) of sham or SMF exposure a second BRS measurement was made (Fig. 2) allowing comparison of the BRS before and after 40 min of sham or SMF exposure. Then MAP, heart rate and MPPG were analyzed. In both series, data obtained between 45 and 50 min (five minutes before $\mathrm{SMF} / \mathrm{sham}$ exposure) were considered as baseline, control values and compared with those obtained after SMF/sham exposure (85-90 min). The flux density of the magnetic field was measured with a Gauss/Tesla meter (Model 4048, F.W. Bell, Division of Bell Technologies; Orlando, FL, USA). The accuracy of the Gaussmeter probe indicated by the manufacturer was $0.01 \mathrm{mT}$. The field intensity without magnetic field exposure did not differ from the intensity of the ambient DC geomagnetic field in the laboratory which was 46-47 $\mu \mathrm{T}$, assessed from the International Geomagnetic Reference Field (IGRF) 1995 model (Data Analysis Center for Geomagnetism and Space Magnetism, Graduate School of Science, Kyoto University, Kyoto, Japan). Data were used only when animals were calm, resting and undisturbed during the entire experimental run (Gmitrov, 2007).

\section{Statistical analysis}

A paired $t$-test or Wilcoxon signed rank test was used, as required, to estimate changes in measured hemodynamic parameters before and after SMF or sham exposure to sinocarotid baroreceptor series (Table 1). One-way ANOVA using a Friedman and Kruskal-Wallis post hoc 
test was then used to compare within-and between-group changes respectively. To estimate SMF effect on BRS - MPPG $\mathrm{Ni}_{\mathrm{Ni}}$, relationship a Pearson or Spearman correlation was used as required. All data are presented as means \pm standard errors of the mean. A $p$ value $<0.05$ was considered as statistically significant.

\section{Results}

A local exposure of the sinocarotid baroreceptors to $350 \mathrm{mT}$ SMF was found to significantly increase $\mathrm{BRS}$ for nitroprusside $\left(\mathrm{BRS}_{\mathrm{Ni}}\right)$ and heart rate variability (Table 1). A notable significant increase of microvascular dilation and blood flow $\left(\mathrm{MPPG}_{\mathrm{Ni}}\right)$ synchronized with increase in heart rate, in response to the same dose and hypotension as control-pre-SMF exposure iv bolus of nitroprusside (Ni3, Ni4 after versus Ni3, Ni4 before SMF exposure) was observed (Figs. 2,3). Additionally, a significant positive correlation between the SMF-induced increase in BRS for nitroprusside and that in microcirculatory blood flow swing up was found $\left(\mathrm{BRS}_{\mathrm{Ni}}\right.$ with $\mathrm{MPPG}_{\mathrm{Ni}}, \mathrm{r}$ $=0.51, \mathrm{n}=24, \mathrm{p}=0.01)$. This was opposite to nitroprusside bolus-induced decrease in MAP, which did not change significantly after SMF exposure (Fig. 3) and show any significant correlation with microvascular blood flow swing up. There was no significant change in baroreflex-mediated hear rate or microcirculatory response after iv bolus injections of phenylephrine. Finally, sham magnets exposures resulted in no effect on BRS, heart rate variability or on the BRS - MPPG relationship (Table 1).

\section{Discussion}

The important and novel findings demonstrated in this study are that arterial baroreflex modulates NO-mediated vasodilation and that sinocarotid baroreceptors stimulation increases vessels sensitivity to NO. This was reflected by a significant positive correlation between improved microvascular dilation $\left(\mathrm{MPPG}_{\mathrm{Ni}}\right)$ and $\mathrm{BRS}$ for nitroprusside $\left(\mathrm{BRS}_{\mathrm{Ni}}\right)$ representing sympathetic, vasoactive arm of the baroreflex response. Enhanced coupling of baroreflex and NO function may trigger a cascade of important cardiovascular mechanisms affecting global hemodynamics. Arterial baroreflex inhibiting intrinsic fluctuations in sympathetic outflow can dynamically sensitize vascular smooth muscle cells to NO, potentiating NO tonic restraint on blood pressure (Gamboa et al., 1912) and the blood pressure buffering system that relies on 
enhanced NO production and vasodilation in response to endothelial shear stress (Stauss and Persson, 2000). On microcirculatory level the same mechanism, coupling neural and endothelial vascular control, may contribute to fine beat-to-beat tuning of microvascular tone adjusted to tissues nutritive requirements, pointing to potential metabolic consequences of microvessels sensitization to NO. Importantly, baroreflex - NO interplay was confirmed in chronic experiments, where sinocarotid baroreceptor SMF exposure decreased blood pressure and evoked an increase in BRS and plasma NO metabolites (Okano and Ohkubo, 2005). A specific inhibitory effect of sympathetic activation on shear-mediated NO release (Hijmering et al., 2002) supplies additional argument for baroreflex-mediated sympathetic activity withdrawal based NOsensitizing vasodilatory mechanism. The depletion of muscle sympathetic nerve traffic by sinocarotid baroreceptors electric stimulation (Gronda et al., 2014) argues for similar sympathoinhibitory effect for magnetic stimulation technique. However, further experiments with chronic SMF sinocarotid baroreceptor exposure and direct measurements of sympathetic activity should be performed to support proposed baroreflex-mediated sympathoinhibitory-NOsensitizing mechanism.

\section{Proposed mechanism of SMF effect on arterial baroreceptors}

A growing body of evidence suggests that mechanosensitive ion channels play a decisive role in magnetoreception. Hughes et al. found that SMF modulated a mechanosensitive ion channel activity in artificial liposomes proposing that the effects of SMFs on ion channels may be mediated by changes in membrane properties due to anisotropic diamagnetism of lipid molecules. Petrov and Martinac supported the above research and found that $400 \mathrm{mT}$ SMF (similar intensity as used in baroreceptor SMF exposure) could reverse gadolinium (Gd) block of the bacterial mechanosensitive channel of large conductance (Hughes et al., 2005, Petrov and Martinac, 2007). The authors suggested that the effect of SMFs may result from changes in physical properties of the lipid bilayer due to diamagnetic anisotropy of phospholipid molecules, which under influence of SMFs could cause displacement of $\mathrm{Gd}^{3+}$ ions from the membrane bilayer and remove the mechanosensitive channel block. However, gadolinium is widely used in baroreceptor research as a non-specific blocker of their activity preventing non-voltage-gated $\mathrm{Ca}^{2+}$ influx through mechanosensitive ion channels during baroreceptor membrane deformation and the process of the mechanoelectrical transduction (Abboud, 2010). These conclusions, taking into account that 
arterial baroreceptors typically belong to the family of mechanoreceptors, profoundly support SMF baroreceptor stimulatory effects giving them a molecular basis mechanism. It is hypothesized, that SMF modulates blood pressure-induced pulsatory changes in lipid bilayer of the baroreceptor membrane, affecting mechanically-gated $\mathrm{Ca}^{2+}$ influx, the process of depolarization and its encoding into action potential discharge, improving mechanosensory transduction and carotid sinus baroreflex hemodynamic responses.

\section{Conclusions and Perspectives}

Presented findings support the concept that the entire arterial system is under baroreflex control and that it is NO-dependent. The amplification of vascular smooth muscle cells sensitivity to NO suggested to be a new mechanism in baroreflex physiology, promotes interactions between sympathetic nervous system and NO (Gamboa et al., 2012), regenerates reduced NO bioavailability a crucial mechanism of microvascular and endothelial dysfunction (Kim et al., 2006; Napoli et al., 2006). The failure of this regulatory mechanism may establish important etiopathogenetic significance in coronary heart disease, arterial hypertension, insulin resistance and diabetes one of which key feature and causing factor is NO deficit and baroreflex dysfunction, importantly appearing in preclinical stages of the disease (Iellamo et al., 2006; Lesniewski et al., 2008). At the same time baroreceptor stimulation provides potential therapeutic strategy for a variety of conditions where sympathovagal imbalance exacerbated by derangements of NO signaling (Schultz, 2009) increases cardiovascular risk substantially (La Rovere et al., 1998; Abboud, 2010; Malpas, 2010). It is speculated that the improvement of vessels sensitivity to NO synergistically reinforce the reciprocal cross-talk relationship between insulin resistance and NO deficit (Kim et al., 2006) arguing for baroreceptor stimulation in cardiometabolic conditions such as diabetes, obesity and the metabolic syndrome where unresponsiveness of vascular smooth muscle cells to NO (more compromised in the microcirculation than in the macrocirculation) amplifies deleterious consequences of reduced endothelial NO availability (Montero et al., 2013). It is not excluded that a high efficiency of sinocarotid baroreceptors electric stimulation in drug resistant arterial hypertension and heart failure (Gronda et al., 2014) employs the same sympathoinhibitory-NO-sensitizing mechanism. However, further research is needed to gain a better understanding of the role of arterial 
baroreflex in the process of vascular tone regulation and potential therapeutic targets of sinocarotid baroreceptors stimulation techniques.

\section{Acknowledgments}

This research was carried out at the Department of Environmental Health in the National Institute of Public Health, Tokyo, Japan, under the auspices of the Japanese Government Research Awards for Foreign Specialists, administrated by the Science and Technology Agency of Japan.

\section{References}

1. Abboud, F.M., 2010. In search of autonomic balance: the good, the bad, and the ugly. Am. J. Physiol., Regul. Integr. Comp. Physiol. 298, R1449-R1467.

2. Bernardi, L., Hayoz, D., Wenzel, R., Passino, C., Calciati, A., Weber, R., Noll, G., 1997. Synchronous and baroreceptor-sensitive oscillations in skin microcirculation: evidence for central autonomic control. Am. J. Physiol. 273, H1867-H1878.

3. Chapleau, M.W., Abboud, F.M., 2004. The baroreceptor reflex: novel methods and mechanisms, in: Dun, N.J., Machado, B.H., Pilowsky, P.M. (Eds.), Neural Mechanisms of Cardiovascular Regulation. Kluwer Academic Publisher, Norwell, Massachusetts, USA.

4. Gamboa, A., Okamoto, L.E., Diedrich, A., Choi, L., Robertson, D., Farley, G., Paranjape, S., Biaggioni, I. 2012. Sympathetic activation and nitric oxide function in early hypertension. Am J Physiol. Heart Circ. Physiol. 302, H1438-H1443. 
5. Gmitrov, J. 2007. Static magnetic field effect on the arterial baroreflex-mediated control of microcirculation: Implications for cardiovascular effects due to environmental magnetic fields. Radiat. Environ. Biophy. 46, 281-290.

6. Gronda, E., Seravalle, G., Brambilla, G., Costantino, G., Casini, A., Alsheraei1, A., Lovett, E.G., Mancia, G., Grassi, G. 2014. Chronic baroreflex activation effects on sympathetic nerve traffic, baroreflex function, and cardiac haemodynamics in heart failure: a proof-of-concept study. Eur. J. Heart Fail. 16, 977-983.

7. Harrison, D.G., Cai, H., 2003. Endothelial control of vasomotion and nitric oxide production. Cardiol. Clin. 21, 289-302.

8. Hijmering, M.L., Stroes, E.S.G., Olijhoek, J., Hutten, B.A., Blankestijn, P.J., Rabelink, T.J., 2002. Sympathetic activation markedly reduces endothelium-dependent, flow-mediated vasodilation. J. Am. Coll. Cardiol. 39, 683-688.

9. Hof, R.P., Takiguchi, Y., Nussberger, J., Hof, A., Kay, M., Peters, T., Zelis, R., Brunner, H.R., 1991. Baroreflex and atrial natriuretic factor concentration correlate with myocardial infarct size and predict early death in rabbits. Implication for drug studies. J. Cardiovasc. Pharm. 18, 361368.

10 Hughes, S,. El., Haj, A.J., Dobson, J., Martinac, B., 2005. The influence of static magnetic fields on mechanosensitive ion channel activity in artificial liposomes. Eur. Biophys. J. 34, 434441.

11. Iellamo, F., Tesauro, M., Rizza, S., Aquilani, S., Cardillo, C., Iantorno, M., Turriziani, M., Lauro, R., 2006. Concomitant impairment in endothelial function and neural cardiovascular regulation in offspring of type 2 diabetic subjects. Hypertension 48, 418-423. 
12. Kim, J., Montagnani, M., Koh, K.K., Quon, M.J., 2006. Reciprocal relationships between insulin resistance and endothelial dysfunction: molecular and pathophysiological mechanisms. Circulation 113, 1888-1904.

13. La Rovere, M.T., Bigger, J.T., Marcus, F.I., Mortara, A., Scwartz, P.J., 1998. Baroreflex sensitivity and heart rate variability in prediction of total cardiac mortality after myocardial infarction. Lancet 351, 478-484.

14. Lesniewski, L.A., Donato, A.J., Behnke, B.J., Woodman, C.R., Laughlin, M.H., Ray, C.A., Delp, M.D., 2008. Decreased NO signaling leads to enhanced vasoconstrictor responsiveness in skeletal muscle arterioles of the ZDF rat prior to overt diabetes and hypertension. J. Physiol. Heart. Circ. Physiol. 294, H1840-H1850.

15. Malpas, S., 2010. Sympathetic nervous system overactivity and its role in the development of cardiovascular disease. Physiol. Rev. 90, 513-557.

16. Montero, D., Walther, G., Pérez-Martin, A., Vicente-Salar, N., Roche, E., Vinet, A., 2013. Vascular smooth muscle function in type 2 diabetes mellitus: a systematic review and metaanalysis. Diabetologia 56, 2122-2133.

17. Napoli, C., de Nigris, F., Williams-Ignarro, S., Pignalosa, O., Sica, V., Ignarro, L.J., 2006. Nitric oxide and atherosclerosis: an update. Nitric Oxide 15, 265-79.

18. Okano, H., Ohkubo, C., 2001. Modulatory effects of static magnetic fields on blood pressure in rabbits. Bioelectromagnetics 22, 408-418.

19. Okano, H., Ohkubo, C., 2005. Exposure to a moderate intensity static magnetic field enhances the hypotensive effect of a calcium channel blocker in spontaneously hypertensive rats. Bioelectromagnetics 26, 611-623. 
20. Omoniyi, A.T., Kett, A., Wu, D., Soong, Y., Clapp, J.F., Szeto, H.H., 2000. A peripheral site of action for the attenuation of baroreflex-mediated bradycardia by intravenous [mu]-opioid agonists. J. Cardiovas. Pharmacol. 35, 269-274.

21. Petrov, E., Martinac, B., 2007. Modulation of channel activity and gadolinium block of MscL by static magnetic fields. Europ. Biophy. J. 36, 95-105.

22. Schröder, H., 2006. No Nitric oxide for HO-1 from sodium nitroprusside. Mol. Pharmacol. 69, 1507-9.

23. Schultz, H.D., 2009. Nitric oxide regulation of autonomic function in heart failure. Curr. Heart Fail. Rep. 6, 71-80.

24. Soares, P.P.S., Ushizima, M.R., Krieger, E.M., Irigoyen, M.C.C., 2005. A semi-automatic computerized method to measure baroreflex-mediated heart rate responses that reduces interobserver variability. Braz. J. Med. Biol. Res. 38, 949-957.

25. Stauss, H.M, Persson, P.B. 2000. Role of nitric oxide in buffering short-term blood pressure fluctuations. News. Physiol. Sci. 15, 229-233.

26. Szili-Torok, T., Paprika, D., Peto, Z., Babik, B., Bari, F., Barzo, P., Rudas, L., 2002. Effect of axillary brachial plexus blockade on baroreflex-induced skin vasomotor responses: assessing the effectiveness of sympathetic blockade. Acta. Anaesthesiol. Scand. 46, 815-820. 


\section{Figures}

Fig. 1 Experimental set-up to explore static magnetic field effect on baroreflex-mediated macro- and microcirculatory response. The right ear including a rabbit's ear chamber (REC) with ingrown skin microvascular networks was positioned under the objective of the microscope for simultaneous intravital observation of the microcirculation and measurement of the microcirculatory blood flow using microphotoelectric plethysmography (MPPG). Another REC is visible on the left free ear. Simultaneously with microcirculatory blood flow femoral artery mean arterial blood pressure (MAP) and heart rate (HR) was measured.

Fig. 2 Upper panel Example of hemodynamic recording following static magnetic field (SMF) local exposure to sinocarotid baroreceptors. MAP, mean femoral arterial blood pressure; HR, heart rate; MPPG, microcirculatory blood flow measured by microphotoelectric plethysmography; BRS, arterial baroreflex sensitivity; SMF “'on', onset of SMF exposure; SMF “'off”, cessation of SMF exposure. (Ni1, 1.0; Ni2, 3.0; Ni3, 10.0; Ni4, 30.0) and (Ph1, 0.3; Ph2, 1.0; $\mathrm{Ph} 3,3.0 ; \mathrm{Ph} 4,10.0)$, doses of sodium nitroprusside and phenylephrine $(\mu \mathrm{g} \mathrm{kg}-1)$, respectively, given by intravenous bolus injection for BRS testing. An increase in baroreflex heart rate and microvasodilatory response on the background of gradual increase in vasodilation (MPPG) and vasomotion (MPPG variability) is obvious.

Lower panel Additional example for experimental run. A notable increase in heart rate variability is apparent.

Fig. 3 Microvasodilatory response to nitroprusside. Segments of five experimental recordings before and after local exposure of sham magnets (control experiment) or $350 \mathrm{mT}$ static magnetic field to sinocarotid baroreceptors. MAP, mean arterial blood pressure; HR, heart rate; MPPG, microcirculatory blood flow measured by microphotoelectric plethysmography; $\mathrm{Ni}, \mathrm{Ph}$, doses of sodium nitroprusside and phenylephrine $\left(\mu \mathrm{g} \mathrm{kg}^{-1}\right)$ given by intravenous bolus injection. CONT, initial control readings; SHAM, after SHAM magnets exposure; SMF, after static magnetic field exposure. A notable increase of the sodium nitroprusside vasodilatory effect (reflected by MPPG swings up) is obvious. 
Fig. 1

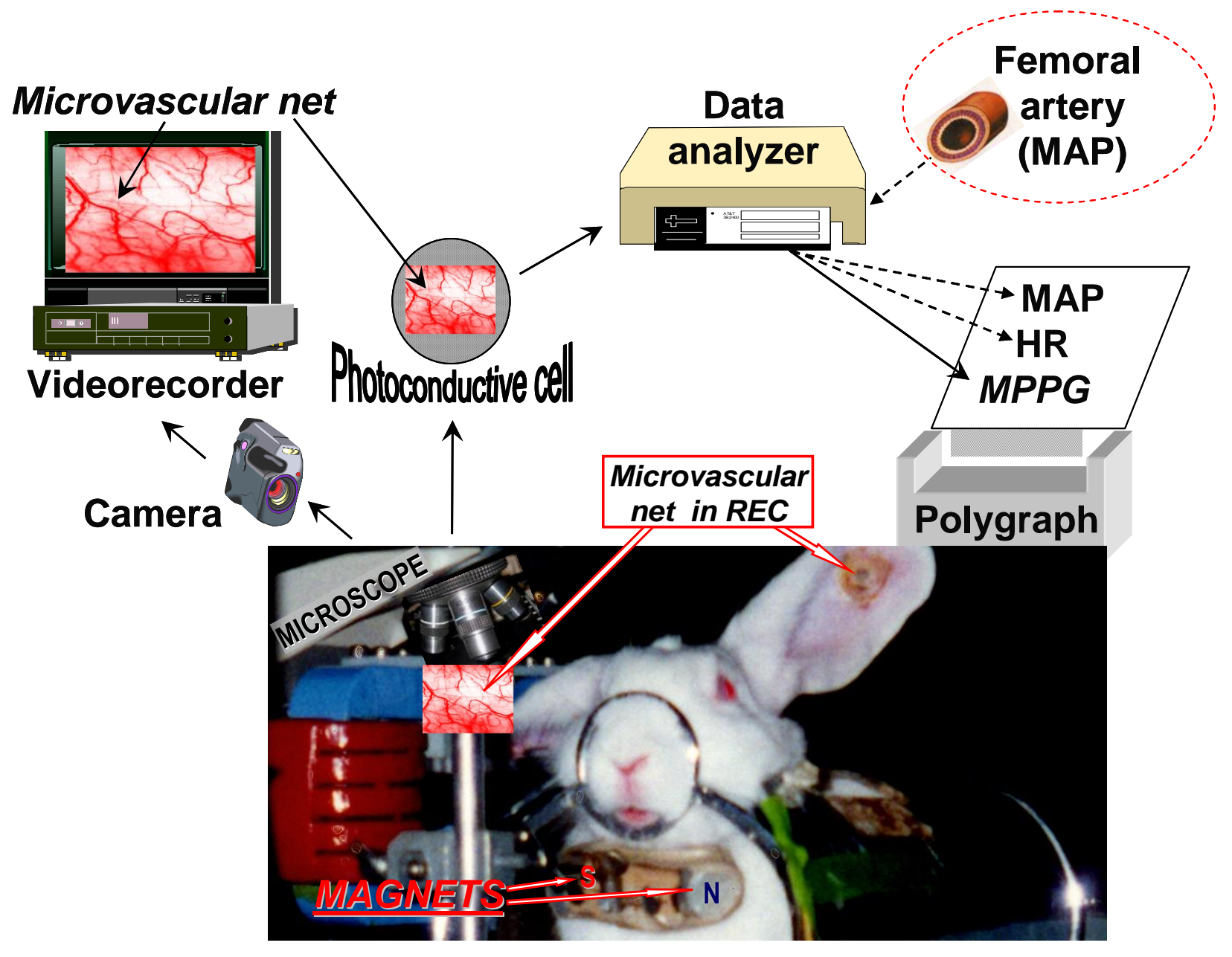


Fig. 2

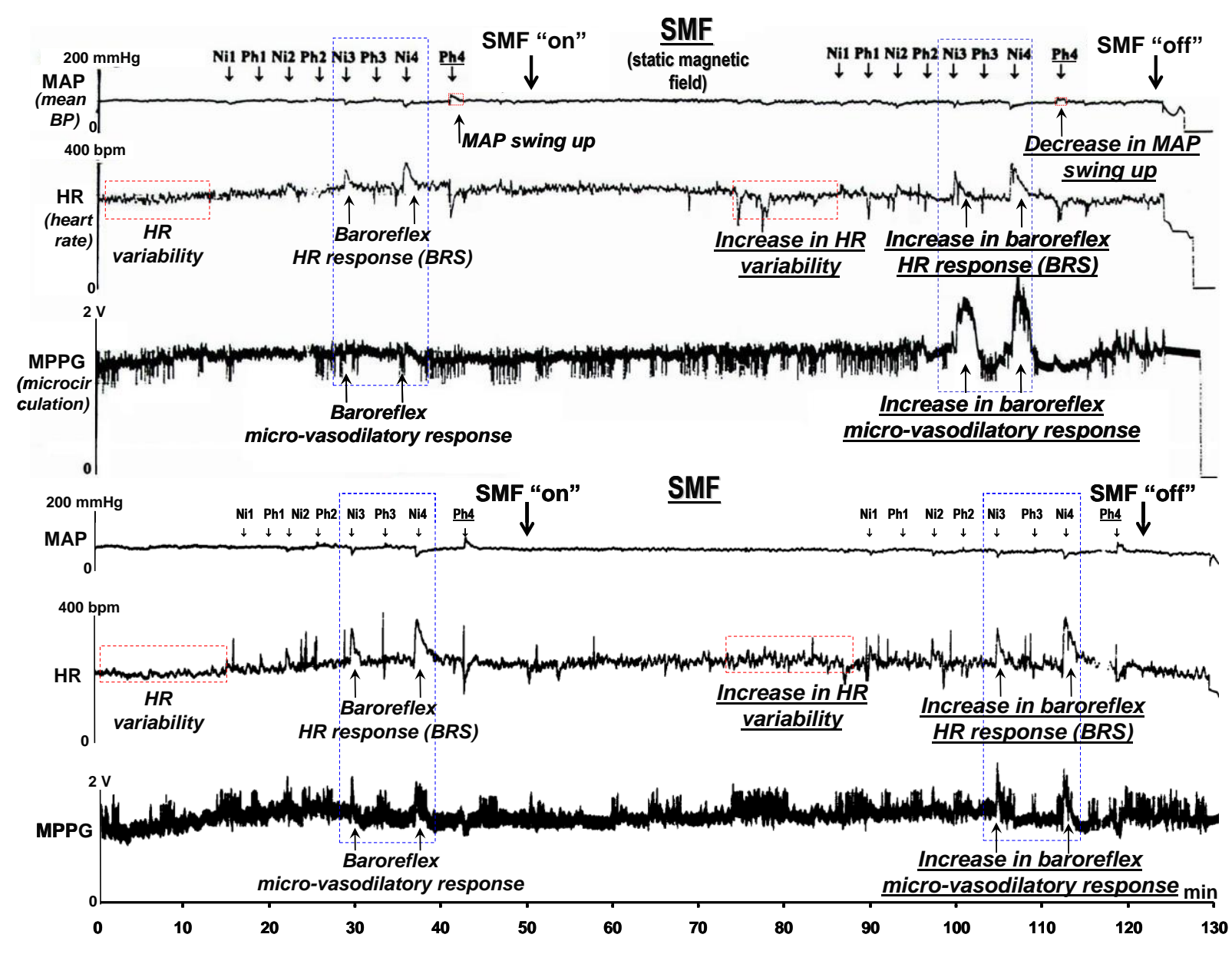


Fig. 3

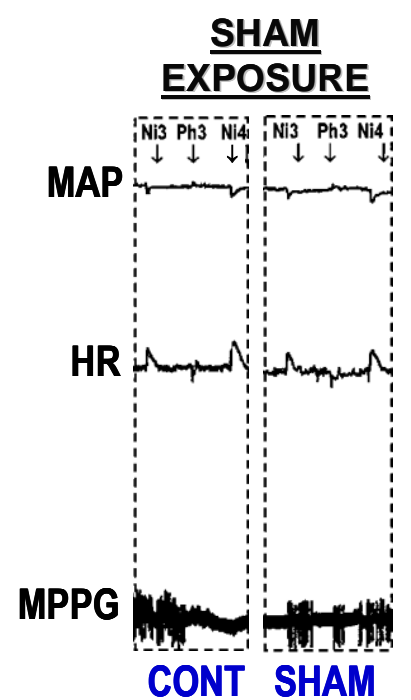

\section{SMF EXPOSURE}

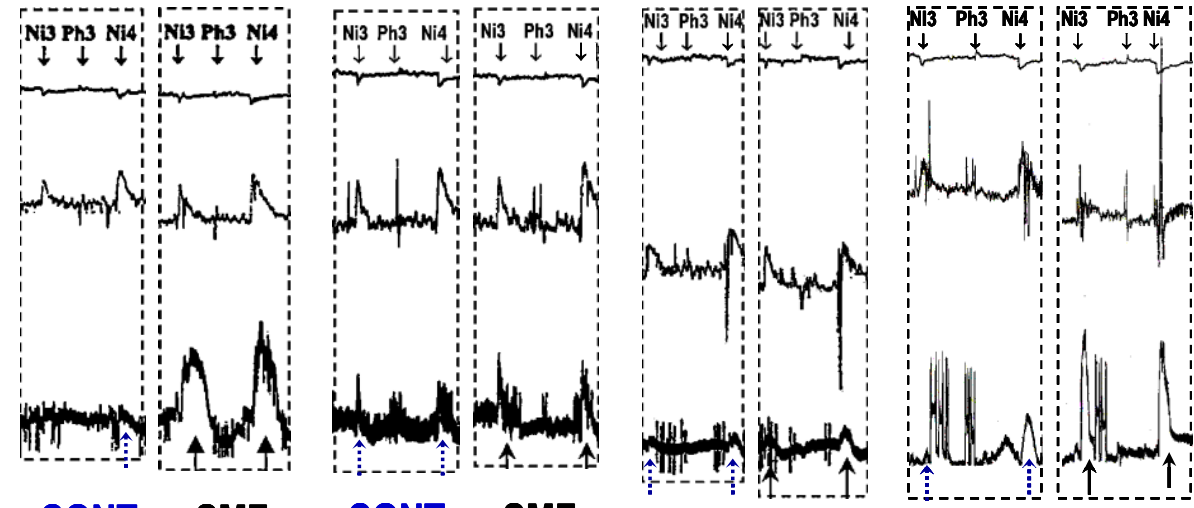

CONT SMF

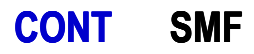

CONT SMF

CONT SMF 
Table 1. Hemodynamic parameters before and after local exposure of sinocarotid baroreceptors

\begin{tabular}{|c|c|c|c|c|c|c|c|}
\hline & $\begin{array}{c}\mathbf{B R S}_{\mathbf{N i}} \\
(\mathrm{bpm} / \mathrm{mmHg})\end{array}$ & $\begin{array}{c}\mathbf{B R S}_{\mathbf{P h}} \\
(\mathrm{bpm} / \mathrm{mmHg})\end{array}$ & $\begin{array}{c}\mathbf{M P P G}_{\mathbf{N i}} \\
(\mathrm{V})\end{array}$ & $\begin{array}{c}\text { MAP } \\
(\mathrm{mmHg})\end{array}$ & $\begin{array}{c}\text { HR } \\
\text { (bpm) }\end{array}$ & $\begin{array}{c}\text { HR SD } \\
(\mathrm{bpm})\end{array}$ & $\begin{array}{c}\text { MPPG } \\
(\mathrm{V})\end{array}$ \\
\hline Control & $\begin{array}{c}\mathbf{3 . 8 0} \pm 0.30 \\
n=24\end{array}$ & $\begin{array}{c}2.74 \pm 0.37 \\
n=14\end{array}$ & $\begin{array}{c}2.57 \pm 0.81 \\
n=24\end{array}$ & $\begin{array}{c}96.3 \pm 2.1 \\
n=8\end{array}$ & $\begin{array}{c}305.8 \pm 10.6 \\
n=8\end{array}$ & $\begin{array}{c}7.7 \pm 0.7 \\
\mathrm{n}=8\end{array}$ & $\begin{aligned} \mathbf{0 . 6 0} & \pm 0.44 \\
n & =7\end{aligned}$ \\
\hline SMF & $\begin{array}{c}4.99 \pm 0.59 \\
n=24 \\
p=0.007\end{array}$ & $\begin{array}{c}3.34 \pm 0.60 \\
n=14 \\
p=0.32\end{array}$ & $\begin{array}{c}\mathbf{7 . 8 2} \pm 1.61 \\
n=24 \\
p<0.0001\end{array}$ & $\begin{array}{c}95.6 \pm 2.3 \\
n=8 \\
p=0.81\end{array}$ & $\begin{array}{c}297.5 \pm 7.8 \\
n=8 \\
p=0.50\end{array}$ & $\begin{array}{c}12.2 \pm 1.7 \\
n=8 \\
p=0.018\end{array}$ & $\begin{array}{c}\mathbf{0 . 5 8} \pm 0.47 \\
n=7 \\
p=0.94\end{array}$ \\
\hline Control & $\begin{array}{c}\mathbf{3 . 5 2} \pm 0.30 \\
\mathrm{n}=24\end{array}$ & $\begin{array}{c}2.90 \pm 0.21 * \\
\mathrm{n}=19\end{array}$ & $\begin{array}{c}\mathbf{0 . 3 3} \pm 0.27 \\
\mathrm{n}=24\end{array}$ & $\begin{array}{c}\mathbf{8 9 . 1} \pm 4.6 \\
n=8\end{array}$ & $\begin{array}{c}288.8 \pm 12.7 \\
n=8\end{array}$ & $\begin{array}{c}14.9 \pm 9.3 \\
\mathrm{n}=8\end{array}$ & $\begin{array}{c}\mathbf{0 . 4 4} \pm 0.78 \\
n=8\end{array}$ \\
\hline SHAM & $\begin{array}{c}2.75 \pm 0.25 \\
n=24 \\
p=0.0013\end{array}$ & $\begin{array}{c}2.67 \pm 0.19 \\
n=19 \\
p=0.20\end{array}$ & $\begin{array}{c}\mathbf{1 . 5 1} \pm 0.99 \\
\mathrm{n}=24 \\
\mathrm{p}=0.43\end{array}$ & $\begin{array}{c}\mathbf{8 8 . 7} \pm 4.2 \\
n=8 \\
p=0.95\end{array}$ & $\begin{array}{c}291.5 \pm 11.7 \\
n=8 \\
p=0.88\end{array}$ & $\begin{array}{c}\mathbf{8 . 8} \pm 1.3 \\
n=8 \\
p=0.16\end{array}$ & $\begin{array}{c}\mathbf{0 . 3 5} \pm 0.75 \\
n=8 \\
p=0.93\end{array}$ \\
\hline
\end{tabular}

The data are mean \pm SEM; $p$, significance. Control, pre exposure values; SMF, after static magnetic field exposure; SHAM, after sham magnets exposure; $\mathrm{BRS}_{\mathrm{Ni}}$, arterial baroreflex sensitivity for nitroprusside; $\mathrm{BRS}_{\mathrm{Ph}}$, arterial baroreflex sensitivity for phenyleprine; MAP, mean arterial blood pressure; HR, heart rate; HR SD, heart rate variability; MPPG, microcirculatory blood flow measured by microphotoelectric plethysmography; $\mathrm{MPPG}_{\mathrm{Ni}}$, microcirculatory blood flow increase following 3,10 and $30 \mu \mathrm{g} \mathrm{kg}^{-1}$ intravenous bolus injections of sodium nitroprusside, $* \mathrm{p}<0.05$. 